\title{
A General Process for the Haloamidation of Olefins. Scope and Mechanism
}

\author{
Ying-Yeung Yeung, Xuri Gao and E. J. Corey* \\ Department of Chemistry and Chemical Biology \\ Harvard University, 12 Oxford Street, Cambridge, Massachusetts 02138
}

$\underline{\text { Supplementary Materials }}$

Materials and Methods. Unless stated otherwise, reactions were performed in flame-dried glassware under a positive pressure of nitrogen using freshly distilled dry solvents. Thin-layer chromatography (TLC) was performed using E. Merck silica gel $60 \mathrm{~F}_{254}$ precoated plates $(0.25$ $\mathrm{mm})$. Flash chromatography was performed using Baker silica gel (40 $\mu \mathrm{m}$ particle size). NMR spectra were recorded on Varian Mercury-300 and Varian Mercury-400 instruments and calibrated using residual undeuterated solvent as an internal reference. IR spectra were recorded on Avatar 360 FT-IR spectrometer. Low-resolution and high-resolution mass spectral analyses were performed at the Harvard University Mass Spectrometry Center. Commercial grade reagents and solvents were used without further purification except as indicated below. Dichloromethane and MeCN were distilled from calcium hydride. DME and THF were distilled from sodium.

General Procedure for Bromoamidation using acetonitrile. To a solution of $\mathrm{N}$ bromoacetamide $(164 \mathrm{mg}, 1.2 \mathrm{mmol})$ in dry acetonitrile $(10 \mathrm{~mL})$ at $0{ }^{\circ} \mathrm{C}$ in dark was added $\mathrm{SnCl}_{4}$ (1.0 $\mathrm{M}$ in $\left.\mathrm{CH}_{2} \mathrm{Cl}_{2}, 0.4 \mathrm{~mL}, 0.4 \mathrm{mmol}\right)$ or $\mathrm{BF}_{3}-\mathrm{Et}_{2} \mathrm{O}\left(1.0 \mathrm{M}\right.$ in $\left.\mathrm{CH}_{2} \mathrm{Cl}_{2}, 0.4 \mathrm{~mL}, 0.4 \mathrm{mmol}\right)$ and 
deionized water $(0.022 \mathrm{~mL}, 1.2 \mathrm{mmol})$ successively. After $5 \mathrm{~min}$, olefin $(1.0 \mathrm{mmol})$ was added at once to the reaction mixture. The reaction was followed by TLC or NMR. After the reaction was complete, saturated $\mathrm{NaHCO}_{3}(3 \mathrm{~mL})$ and $\mathrm{Na}_{2} \mathrm{SO}_{3}(3 \mathrm{~mL})$ were added. The organic layer was separated and the aqueous layer was extracted with $\mathrm{CH}_{2} \mathrm{Cl}_{2}(3 \times 20 \mathrm{~mL})$. The combined extracts were washed with brine $(10 \mathrm{~mL})$, dried $\left(\mathrm{MgSO}_{4}\right)$, filtered and concentrated in vacuo. The residue was purified by flash column chromatography to yield the corresponding haloamide or oxazoline.

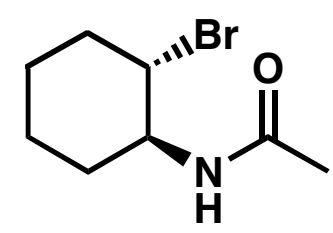

4

$N$-(trans-2-bromocyclohexyl)acetamide (4). White solid; ${ }^{1} \mathrm{H} \mathrm{NMR}\left(400 \mathrm{MHz}, \mathrm{CDCl}_{3}\right) \delta 5.56$ (1H, brs), 3.93 (1H, dddd, $J=10.6,10.6,8.0,4.0 \mathrm{~Hz}), 3.86(1 \mathrm{H}, \mathrm{ddd}, J=10.6,10.6,4.0 \mathrm{~Hz})$, 2.42-2.35 (1H, m), 2.25-2.18 (1H, m), $2.01(3 \mathrm{H}, \mathrm{s}), 1.96-1.86(1 \mathrm{H}, \mathrm{m}), 1.78-1.70(1 \mathrm{H}, \mathrm{m}), 1.50-$ $1.18(4 \mathrm{H}, \mathrm{m}) ;{ }^{13} \mathrm{C} \mathrm{NMR}\left(100 \mathrm{MHz}, \mathrm{CDCl}_{3}\right) \delta 169.8,55.6,55.3,37.3,33.6,26.7,24.5,23.7 ; \mathrm{MS}$ (AP+) $\mathrm{C}_{8} \mathrm{H}_{15} \mathrm{BrNO}\left(\mathrm{MH}^{+}\right) 219.8 ; \mathrm{mp} 122-123^{\circ} \mathrm{C}$. (The physical data were in full accordance with the literature value in all respects: Bellucci, G.; Bianchini, R.; Chiappe, C. J. Org. Chem. 1991, 56, 3067-3073.) 


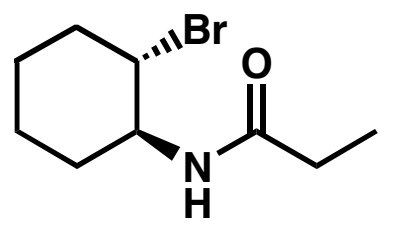

5

$N$-(trans-2-bromocyclohexyl)propionamide (5). To a solution of $N$-bromoacetamide (164 mg, $1.2 \mathrm{mmol})$ in dry propionitrile $(10 \mathrm{~mL})$ at $0{ }^{\circ} \mathrm{C}$ in dark was added $\mathrm{SnCl}_{4}\left(1.0 \mathrm{M}^{\text {in }} \mathrm{CH}_{2} \mathrm{Cl}_{2}, 0.4\right.$ $\mathrm{mL}, 0.4 \mathrm{mmol})$ and deionized water $(0.022 \mathrm{~mL}, 1.2 \mathrm{mmol})$ successively. After $5 \mathrm{~min}$, cyclohexene $(0.1 \mathrm{~mL}, 1.0 \mathrm{mmol})$ was added at once to the reaction mixture. The reaction was followed by NMR. After $1 \mathrm{~h}$, the reaction was quenched with saturated $\mathrm{NaHCO}_{3}(3 \mathrm{~mL})$ and $\mathrm{Na}_{2} \mathrm{SO}_{3}(3 \mathrm{~mL})$. The organic layer was separated and the aqueous layer was extracted with $\mathrm{CH}_{2} \mathrm{Cl}_{2}(3 \times 20 \mathrm{~mL})$. The combined extracts were washed with brine $(10 \mathrm{~mL})$, dried $\left(\mathrm{MgSO}_{4}\right)$, filtered and concentrated in vacuo. The residue was purified by flash column chromatography to yield 5 as a white solid. IR (film) 3246, 2939, 1643, 1558, 1264, $1189 \mathrm{~cm}^{-1}$; ${ }^{1} \mathrm{H}$ NMR (400 MHz, $\left.\mathrm{CDCl}_{3}\right) \delta 5.53(1 \mathrm{H}$, brs $), 3.96(1 \mathrm{H}, \mathrm{dddd}, J=10.8,10.8,7.6,4 . .0 \mathrm{~Hz}), 3.88(1 \mathrm{H}, \mathrm{ddd}, J=10.8$, 10.8, $4.0 \mathrm{~Hz}), 2.42-2.35(1 \mathrm{H}, \mathrm{m}), 2.25-2.18(3 \mathrm{H}, \mathrm{m}), 1.96-1.86(1 \mathrm{H}, \mathrm{m}), 1.78-1.70(1 \mathrm{H}, \mathrm{m}), 1.50-$ $1.20(4 \mathrm{H}, \mathrm{m}), 1.16(3 \mathrm{H}, \mathrm{t}, J=7.6 \mathrm{~Hz}) ;{ }^{13} \mathrm{C} \mathrm{NMR}\left(100 \mathrm{MHz}, \mathrm{CDCl}_{3}\right) \delta 173.4,55.7,55.2,37.3$, 33.6, 30.1, 26.7, 24.6, 10.1; HRMS (ES+) calcd for $\mathrm{C}_{9} \mathrm{H}_{17} \mathrm{BrNO}\left(\mathrm{MH}^{+}\right)$234.0493, found 234.0485; mp 113-114 ${ }^{\circ} \mathrm{C}$.

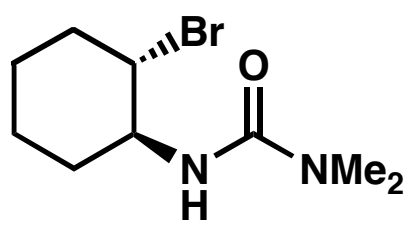


$N^{\prime}$-(trans-2-bromocyclohexyl)- $N, N$-dimethylurea (6). To a solution of $N$-bromoacetamide (164 mg, $1.2 \mathrm{mmol})$ in dry $\mathrm{CH}_{2} \mathrm{Cl}_{2}(2 \mathrm{~mL})$ and $N, N$-dimethylcyanamide $(0.4 \mathrm{~mL}, 5.0 \mathrm{mmol})$ at 0 ${ }^{\circ} \mathrm{C}$ in dark was added $\mathrm{SnCl}_{4}\left(1.0 \mathrm{M}\right.$ in $\left.\mathrm{CH}_{2} \mathrm{Cl}_{2}, 0.4 \mathrm{~mL}, 0.4 \mathrm{mmol}\right)$ and deionized water $(0.022 \mathrm{~mL}$, $1.2 \mathrm{mmol})$ successively. After $5 \mathrm{~min}$, cyclohexene $(0.1 \mathrm{~mL}, 1.0 \mathrm{mmol})$ was added at once to the reaction mixture. After $1 \mathrm{~h}$, the reaction was quenched with saturated $\mathrm{NaHCO}_{3}(3 \mathrm{~mL})$ and $\mathrm{Na}_{2} \mathrm{SO}_{3}(3 \mathrm{~mL})$. The organic layer was separated and the aqueous layer was extracted with $\mathrm{CH}_{2} \mathrm{Cl}_{2}(3 \times 20 \mathrm{~mL})$. The combined extracts were washed with brine $(10 \mathrm{~mL})$, dried $\left(\mathrm{MgSO}_{4}\right)$, filtered and concentrated in vacuo. The residue was purified by flash column chromatography to yield 6 as a white solid. IR (film) 3281, 2936, 1698, 1540, 1391, $1050 \mathrm{~cm}^{-1} ;{ }^{1} \mathrm{H}$ NMR (400 MHz, $\left.\mathrm{CDCl}_{3}\right) \delta 4.52(1 \mathrm{H}, \mathrm{d}, J=6.0 \mathrm{~Hz}), 3.95(1 \mathrm{H}, \mathrm{dt}, J=10.4,4.4 \mathrm{~Hz}), 3.79-3.71(1 \mathrm{H}, \mathrm{m}), 2.90(6 \mathrm{H}$, s), 2.39-2.34 $(1 \mathrm{H}, \mathrm{m}), 2.32-2.25(1 \mathrm{H}, \mathrm{m}), 1.95-1.85(1 \mathrm{H}, \mathrm{m}), 1.74-1.68(2 \mathrm{H}, \mathrm{m}), 1.44-1.19(3 \mathrm{H}$, m); ${ }^{13} \mathrm{C}$ NMR (100 MHz, $\left.\mathrm{CDCl}_{3}\right) \delta$ 158.0, 57.1, 56.8, 37.4, 36.4, 34.3, 26.8, 24.6; HRMS (ES+) calcd for $\mathrm{C}_{9} \mathrm{H}_{18} \mathrm{BrN}_{2} \mathrm{O}\left(\mathrm{MH}^{+}\right)$249.0602, found 249.0610; mp 140-142 ${ }^{\circ} \mathrm{C}$.

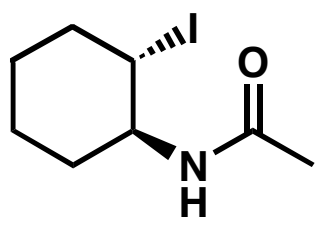

7

$N$-(trans-2-iodocyclohexyl)acetamide (7). To a solution of iodine (254 mg, $1.2 \mathrm{mmol})$ in dry acetonitrile $(10 \mathrm{~mL})$ at $0{ }^{\circ} \mathrm{C}$ in dark was added $\mathrm{BF}_{3}-\mathrm{Et}_{2} \mathrm{O}\left(1.0 \mathrm{M}\right.$ in $\left.\mathrm{CH}_{2} \mathrm{Cl}_{2}, 1.0 \mathrm{~mL}, 1.0 \mathrm{mmol}\right)$ and deionized water $(0.022 \mathrm{~mL}, 1.2 \mathrm{mmol})$ successively. After $5 \mathrm{~min}$, cyclohexene $(0.1 \mathrm{~mL}, 1.0$ mmol) was added at once to the reaction mixture. The reaction was followed by NMR. After 1 $h$, the reaction was quenched with saturated $\mathrm{NaHCO}_{3}(3 \mathrm{~mL})$ and $\mathrm{Na}_{2} \mathrm{SO}_{3}(3 \mathrm{~mL})$. The organic layer was separated and the aqueous layer was extracted with $\mathrm{CH}_{2} \mathrm{Cl}_{2}(3 \times 20 \mathrm{~mL})$. The 
combined extracts were washed with brine $(10 \mathrm{~mL})$, dried $\left(\mathrm{MgSO}_{4}\right)$, filtered and concentrated in vасио. The residue was purified by flash column chromatography to yield 7 as a white solid. IR (film) 3266, 2930, 1649, 1631, 1371, 1168, 1037, $971 \mathrm{~cm}^{-1} ;{ }^{1} \mathrm{H}$ NMR (400 MHz, $\left.\mathrm{CDCl}_{3}\right) \delta 6.39$ $(1 \mathrm{H}, \mathrm{d}, J=8.0 \mathrm{~Hz}), 4.07-3.92(2 \mathrm{H}, \mathrm{m}), 2.50-2.45(1 \mathrm{H}, \mathrm{m}), 2.10-2.02(2 \mathrm{H}, \mathrm{m}), 2.00$ (3H, s), 1.81$1.76(1 \mathrm{H}, \mathrm{m}), 1.56-1.20(4 \mathrm{H}, \mathrm{m}) ;{ }^{13} \mathrm{C} \mathrm{NMR}\left(100 \mathrm{MHz}, \mathrm{CDCl}_{3}\right) \delta$ 169.8, 55.9, 39.7, 36.0, 33.7, 28.1, 24.9, 23.7; HRMS (ES+) calcd for $\mathrm{C}_{8} \mathrm{H}_{15} \mathrm{INO}\left(\mathrm{MH}^{+}\right)$268.0198, found 268.0201; mp 119$121{ }^{\circ} \mathrm{C}$ (lit. $121-123{ }^{\circ} \mathrm{C}$, Radner, F. Acta Chem. Scand. 1989, 43,902-907).

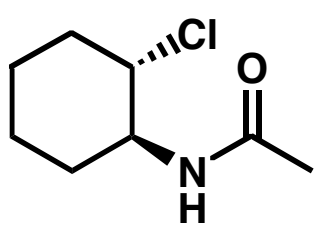

8

$N$-(trans-2-chlorocyclohexyl)acetamide (8). To a solution of $N$-chloroacetamide (112 mg, 1.2 mmol) in dry acetonitrile $(10 \mathrm{~mL})$ at $0{ }^{\circ} \mathrm{C}$ in dark was added $\mathrm{BF}_{3}-\mathrm{Et}_{2} \mathrm{O}\left(1.0 \mathrm{M}\right.$ in $\mathrm{CH}_{2} \mathrm{Cl}_{2}, 1.0 \mathrm{~mL}$, $1.0 \mathrm{mmol})$ and deionized water $(0.022 \mathrm{~mL}, 1.2 \mathrm{mmol})$ successively. After $5 \mathrm{~min}$, cyclohexene $(0.1 \mathrm{~mL}, 1.0 \mathrm{mmol})$ was added at once to the reaction mixture and it was allowed to raise to 23 ${ }^{\circ} \mathrm{C}$. The reaction was followed by NMR. After $20 \mathrm{~h}$, the reaction was quenched with saturated $\mathrm{NaHCO}_{3}(3 \mathrm{~mL})$ and $\mathrm{Na}_{2} \mathrm{SO}_{3}(3 \mathrm{~mL})$. The organic layer was separated and the aqueous layer was extracted with $\mathrm{CH}_{2} \mathrm{Cl}_{2}(3 \times 20 \mathrm{~mL})$. The combined extracts were washed with brine $(10 \mathrm{~mL})$, dried $\left(\mathrm{MgSO}_{4}\right)$, filtered and concentrated in vacuo. The residue was purified by flash column chromatography to yield 8 as a white solid. ${ }^{1} \mathrm{H}$ NMR $\left(300 \mathrm{MHz}, \mathrm{CDCl}_{3}\right) \delta 5.46(1 \mathrm{H}, \mathrm{brs}), 3.86$ $(1 \mathrm{H}, \mathrm{dddd}, J=10.6,10.6,7.5,4.0 \mathrm{~Hz}), 3.72(1 \mathrm{H}, \mathrm{ddd}, J=10.6,10.6,4.0 \mathrm{~Hz}), 2.30-2.13(2 \mathrm{H}, \mathrm{m})$, $2.01(3 \mathrm{H}, \mathrm{s}), 1.82-1.65(2 \mathrm{H}, \mathrm{m}), 1.78-1.70(1 \mathrm{H}, \mathrm{m}), 1.48-1.16(4 \mathrm{H}, \mathrm{m}) ;{ }^{13} \mathrm{C} \mathrm{NMR}(100 \mathrm{MHz}$, $\left.\mathrm{CDCl}_{3}\right) \delta 170.0,62.8,55.2,36.3,33.0,25.6,24.4,23.7 ; \mathrm{MS}(\mathrm{AP}+) \mathrm{C}_{8} \mathrm{H}_{15} \mathrm{ClNO}\left(\mathrm{MH}^{+}\right)$175.8; mp 
$122-124{ }^{\circ} \mathrm{C}$. (The physical data were in full accordance with the literature value in all respects:

Driguez, H.; Paton, J. M.; Lessard, J. Can. J. Chem. 1977, 55, 700-717.)

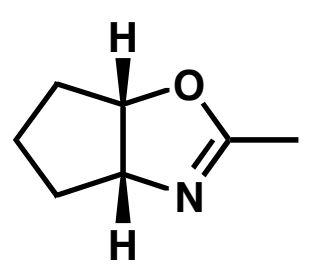

9

cis-3a,5,6,6a-Tetrahydro-2-methyl-4H-cyclopentoxazole (9). Colorless oil; IR (film) 2959, 1668, 1385, 1230, $953 \mathrm{~cm}^{-1} ;{ }^{1} \mathrm{H}$ NMR (400 MHz, $\left.\mathrm{CDCl}_{3}\right) \delta 4.90(1 \mathrm{H}, \mathrm{dd}, J=6.6,6.0 \mathrm{~Hz}), 4.46$ $(1 \mathrm{H}, \mathrm{dd}, J=7.0,6.6 \mathrm{~Hz}), 2.00-1.80(5 \mathrm{H}, \mathrm{m}), 1.66-1.38(4 \mathrm{H}, \mathrm{m}) ;{ }^{13} \mathrm{C} \mathrm{NMR}\left(100 \mathrm{MHz}, \mathrm{CDCl}_{3}\right) \delta$ 164.9, 84.8, 71.6, 34.8, 33.9, 22.3, 13.9; $\mathrm{MS}(\mathrm{AP}+) \mathrm{C}_{7} \mathrm{H}_{13} \mathrm{NO}\left(\mathrm{MH}^{+}\right)$125.8. (The physical data were in full accordance with the literature value in all respects: Hu, N. X.; Aso, Y.; Otsubo, T.; Ogura, F. J. Chem. Soc. Perkin Trans. 1 1989, 1775-1780.)

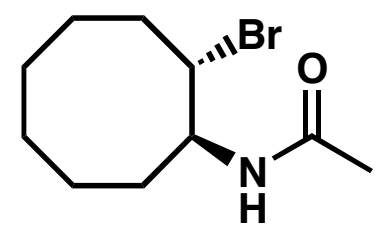

10

$N$-(trans-2-bromocyclooctyl)acetamide (10). Colorless oil. IR (film) 3269, 2929, 1635, 1541, 1369, 1225, $957 \mathrm{~cm}^{-1} ;{ }^{1} \mathrm{H}$ NMR (400 MHz, $\left.\mathrm{CDCl}_{3}\right) \delta, 6.39(1 \mathrm{H}, \mathrm{d}, J=7.6 \mathrm{~Hz}), 4.36-4.30(1 \mathrm{H}, \mathrm{m})$, 3.88-3.80 (1H, m), 2.10-2.04 (2H, m), $1.87(3 \mathrm{H}, \mathrm{s}), 1.73-1.43(10 \mathrm{H}, \mathrm{m}) ;{ }^{13} \mathrm{C}$ NMR (100 MHz, $\left.\mathrm{CDCl}_{3}\right) \delta 169.4,57.1,49.5,34.9,33.5,30.5,29.7,24.5,23.6,23.3$; HRMS (ES+) calcd for $\mathrm{C}_{10} \mathrm{H}_{19} \mathrm{BrNO}\left(\mathrm{MH}^{+}\right)$248.0650, found 248.0643. 


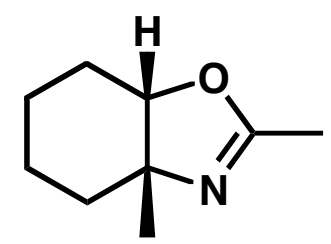

11

cis-4,5,6,7,7a-pentahydro-2,3a-dimethylbenzoxazole (11). Colorless oil. IR (film) 2932, 1658, 1441, 1384, 1229, 1023, $951 \mathrm{~cm}^{-1} ;{ }^{1} \mathrm{H}$ NMR (400 MHz, $\left.\mathrm{CDCl}_{3}\right) \delta 4.84(1 \mathrm{H}, \mathrm{t}, J=4.0 \mathrm{~Hz}), 2.20$ (3H, s), 2.09-1.73 (5H, m), $1.59(3 \mathrm{H}, \mathrm{s}), 1.57-1.42(3 \mathrm{H}, \mathrm{m}) ;{ }^{13} \mathrm{C} \mathrm{NMR}\left(100 \mathrm{MHz}, \mathrm{CDCl}_{3}\right) \delta 175.9$, 91.3, 64.1, 32.1, 25.0, 24.0, 17.5, 16.4, 14.3; HRMS (ES+) calcd for $\mathrm{C}_{9} \mathrm{H}_{16} \mathrm{NO}\left(\mathrm{MH}^{+}\right)$154.1232, found 154.1225.

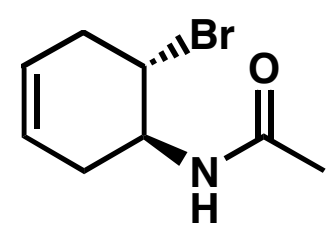

12

$N$-(trans-2-bromo-3-cyclohexenyl)acetamide (12). White solid; IR (film) 3270, 2924, 1648, 1542, 1373, 1312, $733 \mathrm{~cm}^{-1} ;{ }^{1} \mathrm{H}$ NMR (400 MHz, $\left.\mathrm{CDCl}_{3}\right) \delta$ 5.73-5.62 (2H, m), 5.61-5.55 (1H, m), 4.26-4.20 (2H, m), 2.86-2.76 (2H, m), 2.70-2.60 (1H, m), $2.01(3 \mathrm{H}, \mathrm{s}), 2.03-1.99(1 \mathrm{H}, \mathrm{m}) ;{ }^{13} \mathrm{C}$ NMR (100 MHz, $\left.\mathrm{CDCl}_{3}\right) \delta 169.9,124.6,124.5,49.9$, 48.9, 34.1, 30.5, 23.7; HRMS (ES+) calcd for $\mathrm{C}_{8} \mathrm{H}_{13} \mathrm{BrNO}\left(\mathrm{MH}^{+}\right)$218.0180, found 218.0185; mp 86-88 ${ }^{\circ} \mathrm{C}$.

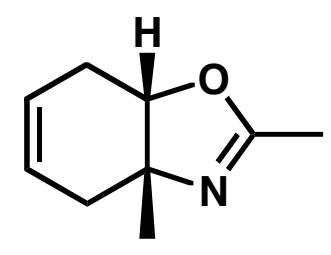

13 
cis-4,7,7a-trihydro-2,3a-dimethylbenzoxazole (13). Colorless oil. IR (film) 2927, 1657, 1546, 1382, 1014, $881 \mathrm{~cm}^{-1} ;{ }^{1} \mathrm{H}$ NMR (400 MHz, $\left.\mathrm{CDCl}_{3}\right) \delta$ 5.93-5.86 (1H, m), 5.83-5.78 (1H, m), 5.10 (1H, brs), $2.75(1 \mathrm{H}, \mathrm{dd}, J=16.4,6.8 \mathrm{~Hz}), 2.62(1 \mathrm{H}, \mathrm{ddd}, J=16.4,6.8,2.0 \mathrm{~Hz}), 2.35(3 \mathrm{H}, \mathrm{s})$, $2.26(1 \mathrm{H}, \mathrm{m}), 1.99-1.94(1 \mathrm{H}, \mathrm{m}), 1.63(3 \mathrm{H}, \mathrm{s}) ;{ }^{13} \mathrm{C} \mathrm{NMR}\left(100 \mathrm{MHz}, \mathrm{CDCl}_{3}\right) \delta$ 175.2, 128.1, 125.1, 91.9, 65.8, 33.0, 27.3, 26.7, 13.6; MS (AP+) $\mathrm{C}_{9} \mathrm{H}_{14} \mathrm{NO}\left(\mathrm{MH}^{+}\right)$151.9.

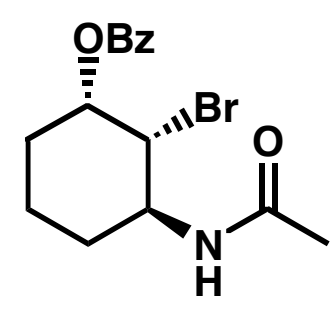

15

$N$-[3-(benzyloxy)-2-bromocyclohexyl]acetamide (15). White solid. IR (film) 3261, 2939, $1718,1652,1268,1026,707 \mathrm{~cm}^{-1} ;{ }^{1} \mathrm{H}$ NMR $\left(400 \mathrm{MHz}, \mathrm{CDCl}_{3}\right) \delta 8.05(2 \mathrm{H}, \mathrm{d}, J=7.6 \mathrm{~Hz}), 7.53$ $(1 \mathrm{H}, \mathrm{t}, J=7.2 \mathrm{~Hz}), 7.42(2 \mathrm{H}, \mathrm{t}, J=7.6 \mathrm{~Hz}), 7.09(1 \mathrm{H}, \mathrm{d}, J=8.4 \mathrm{~Hz}), 5.51(1 \mathrm{H}, \mathrm{brs}), 4.44(1 \mathrm{H}, \mathrm{dq}$, $J=10.0,4.0 \mathrm{~Hz}), 4.26(1 \mathrm{H}, \mathrm{dd}, J=10.0,2.8 \mathrm{~Hz}), 2.21-2.17(1 \mathrm{H}, \mathrm{m}), 2.01(3 \mathrm{H}, \mathrm{s}), 1.75-1.43(5 \mathrm{H}$, m); $\left(400 \mathrm{MHz}, \mathrm{CDCl}_{3}+\mathrm{D}_{2} \mathrm{O}\right) \delta 8.05(2 \mathrm{H}, \mathrm{d}, J=7.6 \mathrm{~Hz}), 7.53(1 \mathrm{H}, \mathrm{t}, J=7.2 \mathrm{~Hz}), 7.42(2 \mathrm{H}, \mathrm{t}, J=$ $7.6 \mathrm{~Hz}), 5.48(1 \mathrm{H}, \mathrm{brs}), 4.40(1 \mathrm{H}, \mathrm{dt}, J=10.0,4.0 \mathrm{~Hz}), 4.23(1 \mathrm{H}, \mathrm{dd}, J=10.0,2.8 \mathrm{~Hz}), 2.21-2.17$ (1H, m), $2.01(3 \mathrm{H}, \mathrm{s}), 1.75-1.43(5 \mathrm{H}, \mathrm{m}) ;{ }^{13} \mathrm{C} \mathrm{NMR}\left(100 \mathrm{MHz}, \mathrm{CDCl}_{3}\right) \delta 170.4,165.9,133.6$, 130.0, 128.8, 128.7, 73.1, 56.2, 51.3, 30.0, 23.7, 19.5; HRMS (ES+) calcd for $\mathrm{C}_{15} \mathrm{H}_{19} \mathrm{BrNO}_{3}$ $\left(\mathrm{MH}^{+}\right) 340.0548$, found $340.0535 ; \mathrm{mp} 145-147{ }^{\circ} \mathrm{C}$. (The corresponding starting material was prepared according to the literature method: Yasui, K.; Fugmi, K.; Tanaka, S.; Tamaru, Y. J. Org. Chem. 1995, 60, 1365-1380.) 


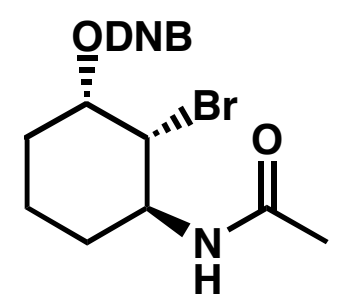

16

$N$-[3-(3,5-dinitrobenzyloxy)-2-bromocyclohexyl]acetamide (16). White solid. IR (film) 3248, 2927, 2845, 1736, 1543, 1347, 1275, 1161, $718 \mathrm{~cm}^{-1} ;{ }^{1} \mathrm{H}$ NMR $\left(400 \mathrm{MHz}, \mathrm{CDCl}_{3}\right) \delta 9.25(1 \mathrm{H}, \mathrm{t}$, $J=2.0 \mathrm{~Hz}), 9.16(2 \mathrm{H}, \mathrm{t}, J=2.0 \mathrm{~Hz}), 5.70(1 \mathrm{H}, \mathrm{d}, J=7.6 \mathrm{~Hz}), 5.61(1 \mathrm{H}, \mathrm{brs}), 4.47(1 \mathrm{H}, \mathrm{dd}, J=$ 9.2, $2.4 \mathrm{~Hz}), 4.30(1 \mathrm{H}, \mathrm{dq}, J=9.2,4.0 \mathrm{~Hz}), 2.35-2.16(2 \mathrm{H}, \mathrm{m}), 2.04(3 \mathrm{H}, \mathrm{s}), 1.85-1.60(4 \mathrm{H}, \mathrm{m})$; $\left(400 \mathrm{MHz}, \mathrm{CDCl}_{3}+\mathrm{D}_{2} \mathrm{O}\right) \delta 9.25(1 \mathrm{H}, \mathrm{t}, J=2.0 \mathrm{~Hz}), 9.16(2 \mathrm{H}, \mathrm{t}, J=2.0 \mathrm{~Hz}), 5.60(1 \mathrm{H}$, brs $), 4.45$ $(1 \mathrm{H}, \mathrm{dd}, J=9.2,2.4 \mathrm{~Hz}), 4.30(1 \mathrm{H}, \mathrm{dt}, J=9.2,4.0 \mathrm{~Hz}), 2.35-2.16(2 \mathrm{H}, \mathrm{m}), 2.04(3 \mathrm{H}, \mathrm{s}), 1.85-$ $1.60(4 \mathrm{H}, \mathrm{m}) ;{ }^{13} \mathrm{C} \mathrm{NMR}\left(100 \mathrm{MHz}, \mathrm{CDCl}_{3}\right) \delta 170.1,161.9,133.8,129.8,123.0,75.0,54.6,52.0$, 29.4, 23.8, 19.7; HRMS (ES+) calcd for $\mathrm{C}_{15} \mathrm{H}_{17} \mathrm{BrN}_{3} \mathrm{O}_{7}\left(\mathrm{MH}^{+}\right)$447.0515, found 447.0502; mp 172-174 ${ }^{\circ} \mathrm{C}$. (The corresponding starting material was prepared according to the literature method: Yasui, K.; Fugmi, K.; Tanaka, S.; Tamaru, Y. J. Org. Chem. 1995, 60, 1365-1380.)

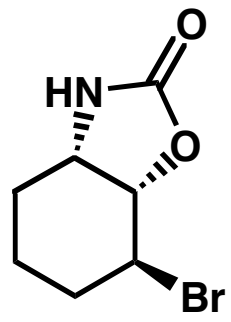

17

4-Bromohexahydro-2(3H)-benzoxoazolinone (17). To a solution of $N$-bromoacetamide (164 $\mathrm{mg}, 1.2 \mathrm{mmol})$ in dry acetonitrile $(10 \mathrm{~mL})$ at $0{ }^{\circ} \mathrm{C}$ in dark was added $\mathrm{SnCl}_{4}\left(1.0 \mathrm{M}^{\text {in }} \mathrm{CH}_{2} \mathrm{Cl}_{2}, 0.1\right.$ $\mathrm{mL}, 0.1 \mathrm{mmol})$ and deionized water $(0.022 \mathrm{~mL}, 1.2 \mathrm{mmol})$ successively. After $5 \mathrm{~min}$, olefin (1.0 
mmol) was added at once to the reaction mixture. The reaction was followed by TLC or NMR. After the reaction finished, it was quenched with saturated $\mathrm{NaHCO}_{3}(3 \mathrm{~mL})$ and $\mathrm{Na}_{2} \mathrm{SO}_{3}(3 \mathrm{~mL})$. The organic layer was separated and the aqueous layer was extracted with $\mathrm{CH}_{2} \mathrm{Cl}_{2}(3 \times 20 \mathrm{~mL})$. The combined extracts were washed with brine $(10 \mathrm{~mL})$, dried $\left(\mathrm{MgSO}_{4}\right)$, filtered and concentrated in vacuo. The residue was purified by flash column chromatography to yield a 1:1 (based on ${ }^{1} \mathrm{H}$ NMR) mixture of $\mathbf{1 7}$ and $\mathbf{2 0}$ as a colorless oil. The mixture was dissolved in $\mathrm{CH}_{2} \mathrm{Cl}_{2}(0.5 \mathrm{~mL})$ and $n$-hexane $(5 \mathrm{~mL})$ and the solution was stirred vigorously for $10 \mathrm{~min}$. The two layers were separated, in which the upper $n$-hexane layer contained 20 and the lower $\mathrm{CH}_{2} \mathrm{Cl}_{2}$ layer contained 17.

17 Colorless crystals. IR (film) 3258, 2943, 1743, 1387, 1026, 903, $726 \mathrm{~cm}^{-1}$; ${ }^{1} \mathrm{H}$ NMR (400 $\left.\mathrm{MHz}, \mathrm{CDCl}_{3}\right) \delta 4.66(1 \mathrm{H}, \mathrm{t}, J=6.0 \mathrm{~Hz}), 4.27(1 \mathrm{H}, \mathrm{m}), 4.04(1 \mathrm{H}, \mathrm{q}, J=5.2 \mathrm{~Hz}), 2.20-2.12(1 \mathrm{H}$, m), 1.95-1.55 (5H, m); ${ }^{13} \mathrm{C}$ NMR (100 $\left.\mathrm{MHz}, \mathrm{CDCl}_{3}\right) \delta 159.8,80.7,52.2,49.2,31.4,27.4,18.8$; MS (AP+) $\mathrm{C}_{9} \mathrm{H}_{14} \mathrm{BrN}_{2} \mathrm{O}_{2}\left(\mathrm{M}+\mathrm{CH}_{3} \mathrm{CN}+\mathrm{H}^{+}\right)$262.9; HRMS (ES+) calcd for $\mathrm{C}_{7} \mathrm{H}_{11} \mathrm{BrNO}_{2}\left(\mathrm{MH}^{+}\right)$ 219.9973, found 219.9966; mp 100-101 ${ }^{\circ} \mathrm{C}$.

$N$-tert-butylacetamide (20) White solid. ${ }^{1} \mathrm{H}$ NMR (400 MHz, $\left.\mathrm{CDCl}_{3}\right) \delta 5.34(1 \mathrm{H}$, brs), $1.90(3 \mathrm{H}$, s), $1.33(1 \mathrm{H}, \mathrm{s}) ;{ }^{13} \mathrm{C} \mathrm{NMR}\left(100 \mathrm{MHz}, \mathrm{CDCl}_{3}\right) \delta 169.7,51.4,29.0,24.7 ; \mathrm{mp} \mathrm{95-97}{ }^{\circ} \mathrm{C}$. (The physical data were in full accordance with the literature value in all respects: Newcomb, M.; Varick, T. R.; Goh, S.-H. J. Am. Chem. Soc. 1990, 5186-5193.)

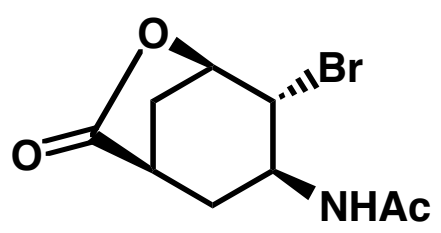

18 
3-Acetylamino-4-bromo-6-oxabicyclo[3.2.1]octan-7-one (18). White solid. IR (film) 3293, 2923, 1765, 1670, 1532, 1225, $967 \mathrm{~cm}^{-1} ;{ }^{1} \mathrm{H} \mathrm{NMR}\left(400 \mathrm{MHz}, \mathrm{CDCl}_{3}\right) \delta 6.38(1 \mathrm{H}, \mathrm{d}, J=6.8 \mathrm{~Hz})$, $4.85(1 \mathrm{H}, \mathrm{dd}, J=5.6,3.6 \mathrm{~Hz}), 4.47(1 \mathrm{H}, \mathrm{brt}, J=6.8 \mathrm{~Hz}), 4.22(1 \mathrm{H}, \mathrm{brs}), 2.60-2.54(2 \mathrm{H}, \mathrm{m}), 2.35-$ $2.30(2 \mathrm{H}, \mathrm{m}), 1.95-1.91(1 \mathrm{H}, \mathrm{m}) 1.88(3 \mathrm{H}, \mathrm{s}) ;{ }^{13} \mathrm{C} \mathrm{NMR}\left(100 \mathrm{MHz}, \mathrm{CDCl}_{3}\right) \delta 178.7,169.6,79.8$, 50.2, 46.5, 35.8, 31.6, 30.0, 23.3; HRMS (ES+) calcd for $\mathrm{C}_{9} \mathrm{H}_{13} \mathrm{BrNO}_{3}\left(\mathrm{MH}^{+}\right)$262.0079, found 262.0087; mp 167-169 ${ }^{\circ} \mathrm{C}$. (The corresponding starting material was prepared according to the literature method: Hatcher, M. A.; Peleg, S.; Dolan, P.; Kensler, T. W.; Sarjeant, A.; Posner, G. H. Bioorg. Med. Chem. 2005, 13, 3964-3976.)

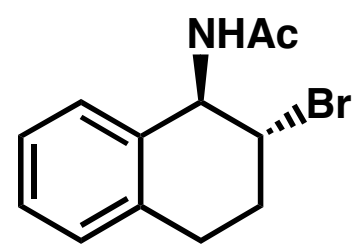

19

N-(trans-2-bromo-1,2,3,4-tetrahydro-1-

naphthalenyl)acetamidecyclohexyl)trimethylacetamide (19). White solid; IR (film) 3264,

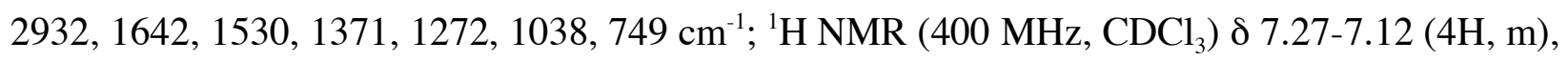
$5.70(1 \mathrm{H}$, brs $), 5.33(1 \mathrm{H}, \mathrm{dd}, J=7.8,4.9 \mathrm{~Hz}), 4.52(1 \mathrm{H}, \mathrm{ddd}, J=5.3,4.9,3.9 \mathrm{~Hz}), 3.10$ (1H, ddd, $J=17.1,8.9,5.9 \mathrm{~Hz}), 2.86(1 \mathrm{H}, \mathrm{ddd}, J=17.1,5.4,5.4 \mathrm{~Hz}), 2.36-2.21(2 \mathrm{H}, \mathrm{m}), 2.03(3 \mathrm{H}, \mathrm{s}) ;{ }^{13} \mathrm{C}$ NMR (100 MHz, $\left.\mathrm{CDCl}_{3}\right) \delta 169.4,135.8,133.5,129.8,129.2,128.3,127.0,54.5,51.0,28.3,26.6$, 23.5; HRMS (ES+) calcd for $\mathrm{C}_{12} \mathrm{H}_{15} \mathrm{BrNO}\left(\mathrm{MH}^{+}\right)$268.0337, found 268.0327; mp 132-133 ${ }^{\circ} \mathrm{C}$.

General Procedure for Bromoamidation using various nitriles. To a solution of $N$ bromoacetamide $(164 \mathrm{mg}, 1.2 \mathrm{mmol})$ in dry $\mathrm{CH}_{2} \mathrm{Cl}_{2}(2 \mathrm{~mL})$ and nitrile $(15 \mathrm{mmol})$ at $0{ }^{\circ} \mathrm{C}$ in dark 
was added $\mathrm{BF}_{3}-\mathrm{Et}_{2} \mathrm{O}\left(1.0 \mathrm{M}\right.$ in $\left.\mathrm{CH}_{2} \mathrm{Cl}_{2}, 0.4 \mathrm{~mL}, 0.4 \mathrm{mmol}\right)$ and deionized water $(0.022 \mathrm{~mL}, 1.2$ mmol) successively. After $5 \mathrm{~min}$, cyclohexene $(0.1 \mathrm{~mL}, 1.0 \mathrm{mmol})$ was added at once to the reaction mixture. The reaction was followed by NMR. After the reaction finished, it was quenched with saturated $\mathrm{NaHCO}_{3}(3 \mathrm{~mL})$ and $\mathrm{Na}_{2} \mathrm{SO}_{3}(3 \mathrm{~mL})$. The organic layer was separated and the aqueous layer was extracted with $\mathrm{CH}_{2} \mathrm{Cl}_{2}(3 \times 20 \mathrm{~mL})$. The combined extracts were washed with brine $(10 \mathrm{~mL})$, dried $\left(\mathrm{MgSO}_{4}\right)$, filtered and concentrated in vacuo. The residue was purified by flash column chromatography to yield the corresponding bromoamide.

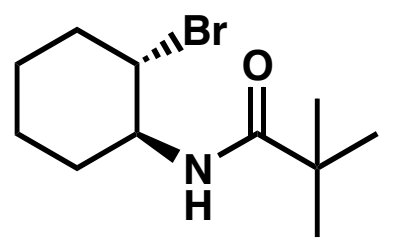

$21 \mathrm{a}$

$N$-(trans-2-bromocyclohexyl)trimethylacetamide (21a). White solid; IR (film) 3297, 2939, 1631, 1542, 1321, 1197, $953 \mathrm{~cm}^{-1} ;{ }^{1} \mathrm{H}$ NMR (400 MHz, $\left.\mathrm{CDCl}_{3}\right) \delta 5.62(1 \mathrm{H}, \mathrm{brs}), 3.96-3.82(2 \mathrm{H}$, m), 2.42-2.35 (1H, m), 2.22-2.18 (1H, m), 1.96-1.86 (1H, m), 1.78-1.70 (1H, m), 1.50-1.18 (4H, m), $1.20(9 \mathrm{H}, \mathrm{m}) ;{ }^{13} \mathrm{C} \mathrm{NMR}\left(100 \mathrm{MHz}, \mathrm{CDCl}_{3}\right) \delta$ 178.0, 55.8, 55.3, 39.0, 37.4, 33.5, 27.8, 26.8, 24.6; HRMS (ES+) calcd for $\mathrm{C}_{11} \mathrm{H}_{21} \mathrm{BrNO}\left(\mathrm{MH}^{+}\right)$262.0806, found 262.0800; $\mathrm{mp} 123-124^{\circ} \mathrm{C}$.

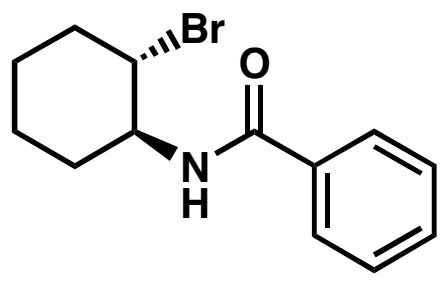

21b

$N$-(trans-2-bromocyclohexyl)benzamide (21b). To a solution of $N$-bromoacetamide (164 mg, $1.2 \mathrm{mmol})$ in dry benzonitrile $(2 \mathrm{~mL})$ at $0{ }^{\circ} \mathrm{C}$ in dark was added $\mathrm{BF}_{3}-\mathrm{Et}_{2} \mathrm{O}\left(1.0 \mathrm{M}\right.$ in $\mathrm{CH}_{2} \mathrm{Cl}_{2}, 0.4$ 
$\mathrm{mL}, 0.4 \mathrm{mmol})$ and deionized water $(0.022 \mathrm{~mL}, 1.2 \mathrm{mmol})$ successively. After $5 \mathrm{~min}$, cyclohexene $(0.1 \mathrm{~mL}, 1.0 \mathrm{mmol})$ was added at once to the reaction mixture. After $1 \mathrm{~h}$, the reaction was quenched with saturated $\mathrm{NaHCO}_{3}(3 \mathrm{~mL})$ and $\mathrm{Na}_{2} \mathrm{SO}_{3}(3 \mathrm{~mL})$. The organic layer was separated and the aqueous layer was extracted with $\mathrm{CH}_{2} \mathrm{Cl}_{2}(3 \times 20 \mathrm{~mL})$. The combined extracts were washed with brine $(10 \mathrm{~mL})$, dried $\left(\mathrm{MgSO}_{4}\right)$, filtered and concentrated in vacuo. The residue was purified by flash column chromatography to yield $\mathbf{2 1 b}$ as a white solid. IR (film) $3219,2934,1632,1547,1236,1192,702 \mathrm{~cm}^{-1} ;{ }^{1} \mathrm{H}$ NMR (400 MHz, $\left.\mathrm{CDCl}_{3}\right) \delta$ 7.82-7.78 $(2 \mathrm{H}, \mathrm{m}), 7.56-7.42(3 \mathrm{H}, \mathrm{m}), 6.16(1 \mathrm{H}, \mathrm{brs}), 4.13(1 \mathrm{H}, \mathrm{dddd}, J=10.6,10.6,8.0,4.0 \mathrm{~Hz}), 4.03(1 \mathrm{H}$, ddd, $J=10.6,10.6,4.0 \mathrm{~Hz}), 2.48-2.32(2 \mathrm{H}, \mathrm{m}), 2.03-1.92(1 \mathrm{H}, \mathrm{m}), 1.83-1.75(2 \mathrm{H}, \mathrm{m}), 1.56-1.45$ (1H, m), 1.42-1.30 (2H, m); ${ }^{13} \mathrm{C}$ NMR (100 MHz, $\left.\mathrm{CDCl}_{3}\right) \delta 167.3,135.0,131.7,128.8,127.2$, 56.0, 55.6, 37.4, 33.6, 26.8, 24.6; HRMS (ES+) calcd for $\mathrm{C}_{13} \mathrm{H}_{17} \mathrm{BrNO}\left(\mathrm{MH}^{+}\right)$282.0493, found 282.0504; mp 140-141 ${ }^{\circ} \mathrm{C}$.

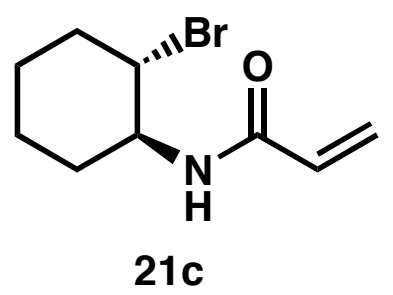

$N$-(trans-2-bromocyclohexyl)acrylamide (21c). White solid. IR (film) 3220, 2963, 1657, 1234, 1187, $1035 \mathrm{~cm}^{-1} ;{ }^{1} \mathrm{H}$ NMR $\left(400 \mathrm{MHz}, \mathrm{CDCl}_{3}\right) \delta 6.29(1 \mathrm{H}, \mathrm{dd}, J=16.8,0.8 \mathrm{~Hz}), 6.15(1 \mathrm{H}, \mathrm{d}, J=$ $10.4 \mathrm{~Hz}), 6.11(1 \mathrm{H}, \mathrm{d}, J=10.4 \mathrm{~Hz}), 6.08(1 \mathrm{H}, \mathrm{brs}), 5.64(1 \mathrm{H}, \mathrm{dd}, J=10.4,1.2 \mathrm{~Hz}), 4.07-3.98$ $(1 \mathrm{H}, \mathrm{m}), 3.93(1 \mathrm{H}, \mathrm{dt}, J=10.8,4.4 \mathrm{~Hz}), 2.40-2.36(1 \mathrm{H}, \mathrm{m}), 2.23-2.19(1 \mathrm{H}, \mathrm{m}), 1.96-1.72(3 \mathrm{H}$, m), 1.49-1.22 (3H, m); $\left.{ }^{13} \mathrm{C} \mathrm{NMR} \mathrm{(100} \mathrm{MHz,} \mathrm{CDCl}_{3}\right) \delta$ 165.2, 131.2, 126.9, 55.4, 37.3, 33.5, 26.7, 24.5; HRMS (ES+) calcd for $\mathrm{C}_{9} \mathrm{H}_{15} \mathrm{BrNO}\left(\mathrm{MH}^{+}\right)$232.0337, found 232.0335; mp 136-138 ${ }^{\circ} \mathrm{C}$. 


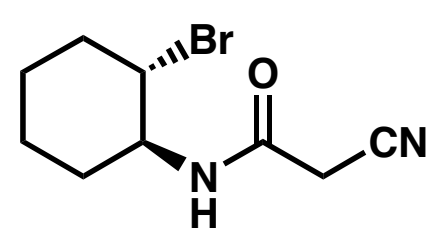

21d

$N$-(trans-2-bromocyclohexyl)cyanoacetamide (21d). White solid; IR (film) 3284, 2941, 2263, $2214,1658,1544,1348,1176 \mathrm{~cm}^{-1} ;{ }^{1} \mathrm{H}$ NMR $\left(400 \mathrm{MHz}, \mathrm{CDCl}_{3}\right) \delta 6.22(1 \mathrm{H}, \mathrm{brs}), 4.02-3.86(2 \mathrm{H}$, m), $3.41(2 \mathrm{H}, \mathrm{s}), 2.45-2.35(1 \mathrm{H}, \mathrm{m}), 2.22-2.13(1 \mathrm{H}, \mathrm{m}), 2.01(3 \mathrm{H}, \mathrm{s}), 1.96-1.85(1 \mathrm{H}, \mathrm{m}), 1.82-$ $1.72(2 \mathrm{H}, \mathrm{m}), 1.50-1.25(3 \mathrm{H}, \mathrm{m}) ;{ }^{13} \mathrm{C} \mathrm{NMR}\left(100 \mathrm{MHz}, \mathrm{CDCl}_{3}\right) \delta 160.6,114.9,56.5,54.7,37.4$, 33.3, 26.7, 26.3, 24.5; HRMS (ES+) calcd for $\mathrm{C}_{11} \mathrm{H}_{17} \mathrm{BrN}_{3} \mathrm{O}\left(\mathrm{M}+\mathrm{CH}_{3} \mathrm{CN}+\mathrm{H}^{+}\right)$286.0555, found 286.0566; mp 129-130 ${ }^{\circ} \mathrm{C}$.

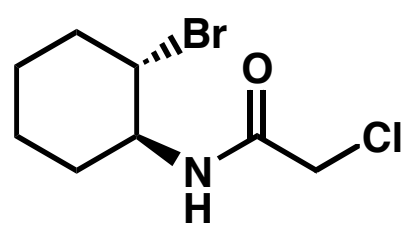

21e

$N$-(trans-2-bromocyclohexyl)chloroacetamide (21e). White solid; IR (film) 3274, 2936, 1640, 1539, 1326, 1195, $775 \mathrm{~cm}^{-1} ;{ }^{1} \mathrm{H}$ NMR (400 MHz, $\left.\mathrm{CDCl}_{3}\right) \delta 6.60(1 \mathrm{H}$, brs), $4.03(2 \mathrm{H}, \mathrm{ABq}, J=$ $15.0 \mathrm{~Hz}), 4.02-3.88(2 \mathrm{H}, \mathrm{m}), 2.42-2.35(1 \mathrm{H}, \mathrm{m}), 2.23-2.16(1 \mathrm{H}, \mathrm{m}), 1.96-1.86(1 \mathrm{H}, \mathrm{m}), 1.80-1.72$ $(2 \mathrm{H}, \mathrm{m}), 1.50-1.26(3 \mathrm{H}, \mathrm{m}) ;{ }^{13} \mathrm{C} \mathrm{NMR}\left(100 \mathrm{MHz}, \mathrm{CDCl}_{3}\right) \delta 165.5,55.8,54.7,42.9,37.2,33.2$, 26.6, 24.5; HRMS (ES+) calcd for $\mathrm{C}_{8} \mathrm{H}_{14} \mathrm{ClBrNO}\left(\mathrm{MH}^{+}\right)$253.9947, found 253.9951; mp 127$128^{\circ} \mathrm{C}$.

General Procedure for the Synthesis of Aziridines. To a solution of bromoamide ( $0.1 \mathrm{mmol})$ in dry THF $(2 \mathrm{~mL})$ at $0{ }^{\circ} \mathrm{C}$ was added at once lithium hexamethyldisilazane (LiHMDS) $(1.0 \mathrm{M}$ in 
THF, $0.12 \mathrm{~mL}, 0.12 \mathrm{mmol}$ ). After $30 \mathrm{~min}$, the reaction was quenched with $\mathrm{pH} 7$ buffer solution (potassium phosphate monobasic sodium hydroxide, $0.2 \mathrm{~mL}$ ). The organic layer was separated and the aqueous layer was extracted with $\mathrm{Et}_{2} \mathrm{O}(3 \times 2 \mathrm{~mL})$. The combined extracts were washed with brine $(0.5 \mathrm{~mL})$, dried $\left(\mathrm{MgSO}_{4}\right)$, filtered and concentrated in vacuo. The residue was purified by flash column chromatography on a short plug of silica gel to yield the corresponding aziridine.

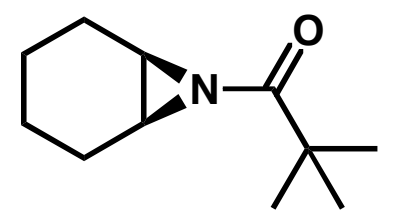

22a

$N$-Pivaloyl-7-azabicyclo[4.1.0.]heptane (22a). Colorless oil. IR (film) 2931, 1680, 1411, 1288, 1191, $1149 \mathrm{~cm}^{-1} ;{ }^{1} \mathrm{H}$ NMR (400 MHz, $\left.\mathrm{CDCl}_{3}\right) \delta$ 2.64-2.61 (2H, m), 1.96-1.89 (2H, m), 1.82-1.78 $(2 \mathrm{H}, \mathrm{m}), 1.44-1.40(4 \mathrm{H}, \mathrm{m}), 1.28-1.24(4 \mathrm{H}, \mathrm{m}), 1.23(9 \mathrm{H}, \mathrm{s}) ;{ }^{13} \mathrm{C} \mathrm{NMR}\left(100 \mathrm{MHz}, \mathrm{CDCl}_{3}\right) \delta$ 192.7, 41.4, 36.1, 28.3, 23.9, 20.2; HRMS (ES+) calcd for $\mathrm{C}_{11} \mathrm{H}_{20} \mathrm{NO}\left(\mathrm{MH}^{+}\right)$182.1545, found 182.1548. (The physical data were in full accordance with the literature value in all respects: Mordini, A.; Russo, F.; Valacchi, M.; Zani, L.; Degl'Innocenti, A.; Reginato, G. Tetrahedron 2002, 58, 7153-7163.)

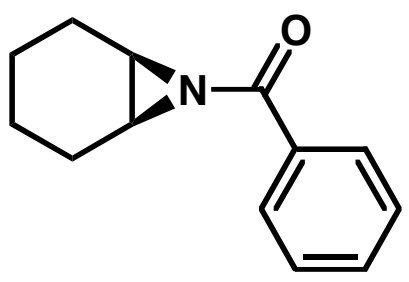

22b 
$N$-Phenyl-7-azabicyclo[4.1.0.]heptane (22b). Colorless oil. IR (film) 2924, 1667, 1409, 11285, 1227, 1115, $703 \mathrm{~cm}^{-1} ;{ }^{1} \mathrm{H}$ NMR $\left(400 \mathrm{MHz}, \mathrm{CDCl}_{3}\right) \delta 7.98(2 \mathrm{H}, \mathrm{d}, J=8.0 \mathrm{~Hz}), 7.53(1 \mathrm{H}, \mathrm{t}, J=$ $7.3 \mathrm{~Hz}), 7.44(2 \mathrm{H}, \mathrm{t}, J=7.6 \mathrm{~Hz}), 2.75(2 \mathrm{H}, \mathrm{m}), 2.11-2.04(2 \mathrm{H}, \mathrm{m}), 1.94-1.88(2 \mathrm{H}, \mathrm{m}), 1.58-1.53$ (2H, m), 1.37-1.34 (2H, m); $\left.{ }^{13} \mathrm{C} \mathrm{NMR} \mathrm{(100} \mathrm{MHz,} \mathrm{CDCl}_{3}\right) \delta$ 180.5, 133.9, 132.7, 129.3, 128.6, 37.3, 24.2, 20.2; HRMS (ES+) calcd for $\mathrm{C}_{13} \mathrm{H}_{16} \mathrm{NO}\left(\mathrm{MH}^{+}\right)$202.1232, found 202.1240.

General Procedure for the Synthesis of Oxazolines. To a solution of bromoamide ( $0.3 \mathrm{mmol})$ in dry DME $(1 \mathrm{~mL})$ was added $\mathrm{Et}_{3} \mathrm{~N}(0.08 \mathrm{~mL}, 0.6 \mathrm{mmol})$ and 1,8-diazabicyclo[5.4.0]undec-7ene (DBU) $(0.01 \mathrm{~mL}, 0.03 \mathrm{mmol})$ successively. The resultant solution was heated to reflux for 4 h. After cooling down to room temperature, the salt was filtered and washed thoroughly with ethyl acetate. The filtrate was concentrated in vacuo and the residue was purified by flash column chromatography on a short plug of silica gel to yield the corresponding oxazoline.

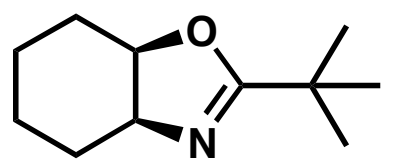

23a

cis-3a,4,5,6,7,7a-Hexahydro-2-tert-butylbenzoxazole (23a). Colorless oil. IR (film) 2933, 1646, 1457, 1137, 1098, 973, $920 \mathrm{~cm}^{-1} ;{ }^{1} \mathrm{H}$ NMR $\left(400 \mathrm{MHz}, \mathrm{CDCl}_{3}\right) \delta 4.42(1 \mathrm{H}, \mathrm{dt}, J=5.2,10.0$ $\mathrm{Hz}), 3.85(1 \mathrm{H}, \mathrm{dt}, J=5.6,8.0 \mathrm{~Hz}), 1.79-1.66(4 \mathrm{H}, \mathrm{m}), 1.53-1.27(4 \mathrm{H}, \mathrm{m}), 1.17(9 \mathrm{H}, \mathrm{s}) ;{ }^{13} \mathrm{C} \mathrm{NMR}$ $\left(100 \mathrm{MHz}, \mathrm{CDCl}_{3}\right) \delta 174.5,78.5,63.0,33.3,27.8,27.6,26.2,19.6,19.0$; HRMS (ES+) calcd for $\mathrm{C}_{11} \mathrm{H}_{20} \mathrm{NO}\left(\mathrm{MH}^{+}\right)$182.1545, found 182.1549 . 


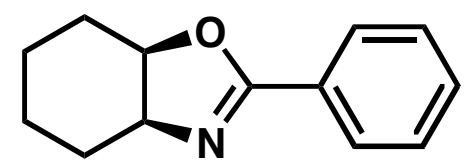

23b

cis-3a,4,5,6,7,7a-Hexahydro-2-phenylbenzoxazole (23b). Colorless oil. IR (film) 2933, 1637, 1448, 1345, 1061, 971, 774, $694 \mathrm{~cm}^{-1} ;{ }^{1} \mathrm{H}$ NMR $\left(400 \mathrm{MHz}, \mathrm{CDCl}_{3}\right) \delta 7.94(2 \mathrm{H}, \mathrm{d}, J=7.2 \mathrm{~Hz})$, $7.45(1 \mathrm{H}, \mathrm{t}, J=7.6 \mathrm{~Hz}), 7.38(2 \mathrm{H}, \mathrm{t}, J=8.0 \mathrm{~Hz}), 4.66(1 \mathrm{H}, \mathrm{dt}, J=5.2,8.0 \mathrm{~Hz}), 4.10(1 \mathrm{H}, \mathrm{dt}, J=$ 6.0, 8.0 Hz),1.94-1.78 (4H, m), 1.66-1.33 (4H, m); $\left.{ }^{13} \mathrm{C} \mathrm{NMR} \mathrm{(100} \mathrm{MHz,} \mathrm{CDCl}_{3}\right) \delta$ 164.4, 131.4, $128.6,128.5,128.3,79.0,63.8,27.9,26.4,20.1,19.3$. (The physical data were in full accordance with the literature value in all respects: Hu, N. X.; Aso, Y.; Otsubo, T.; Ogura, F. J. Chem. Soc. Perkin Trans. I 1989, 1775-1780.) 


\section{Part II X-ray Structure}

X-ray Structure of (18)

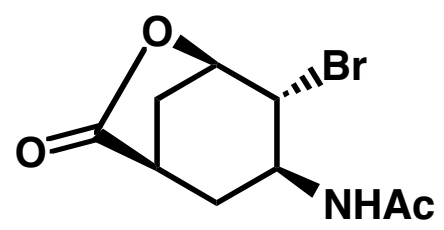

18

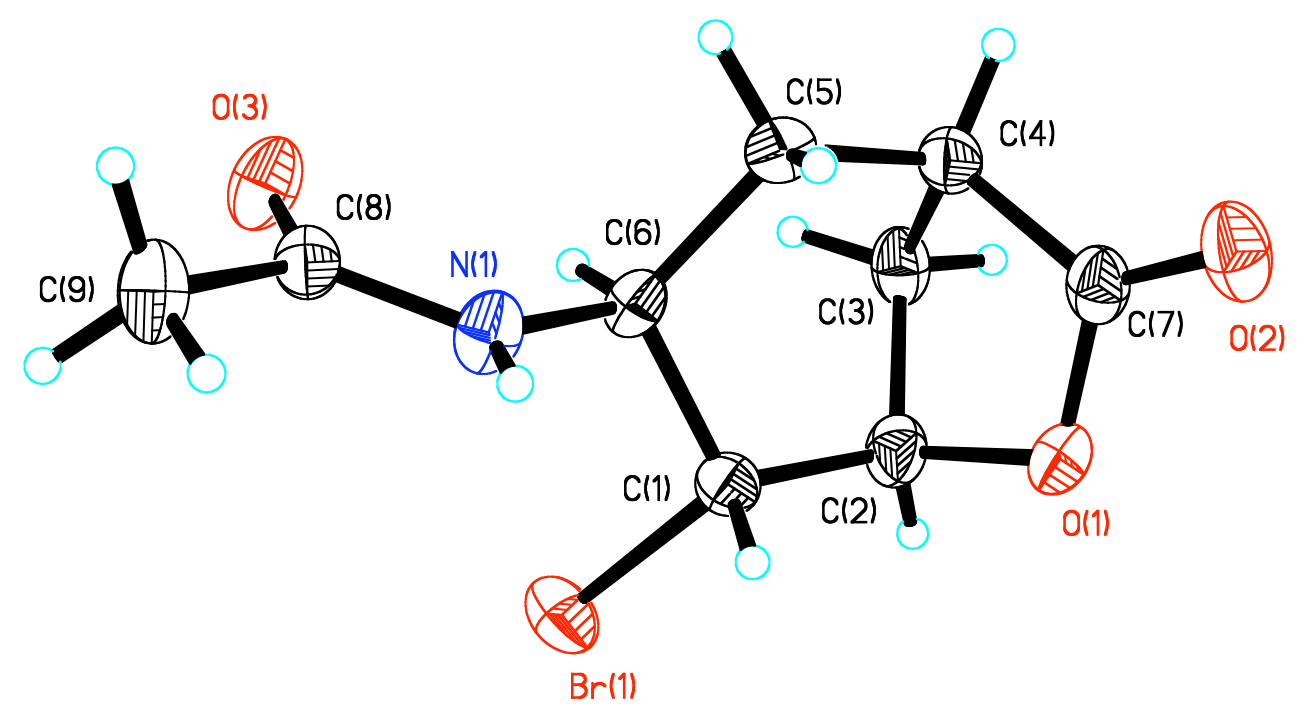


X-ray Structure of (17)

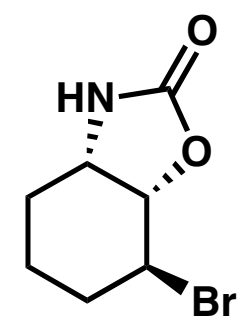

17

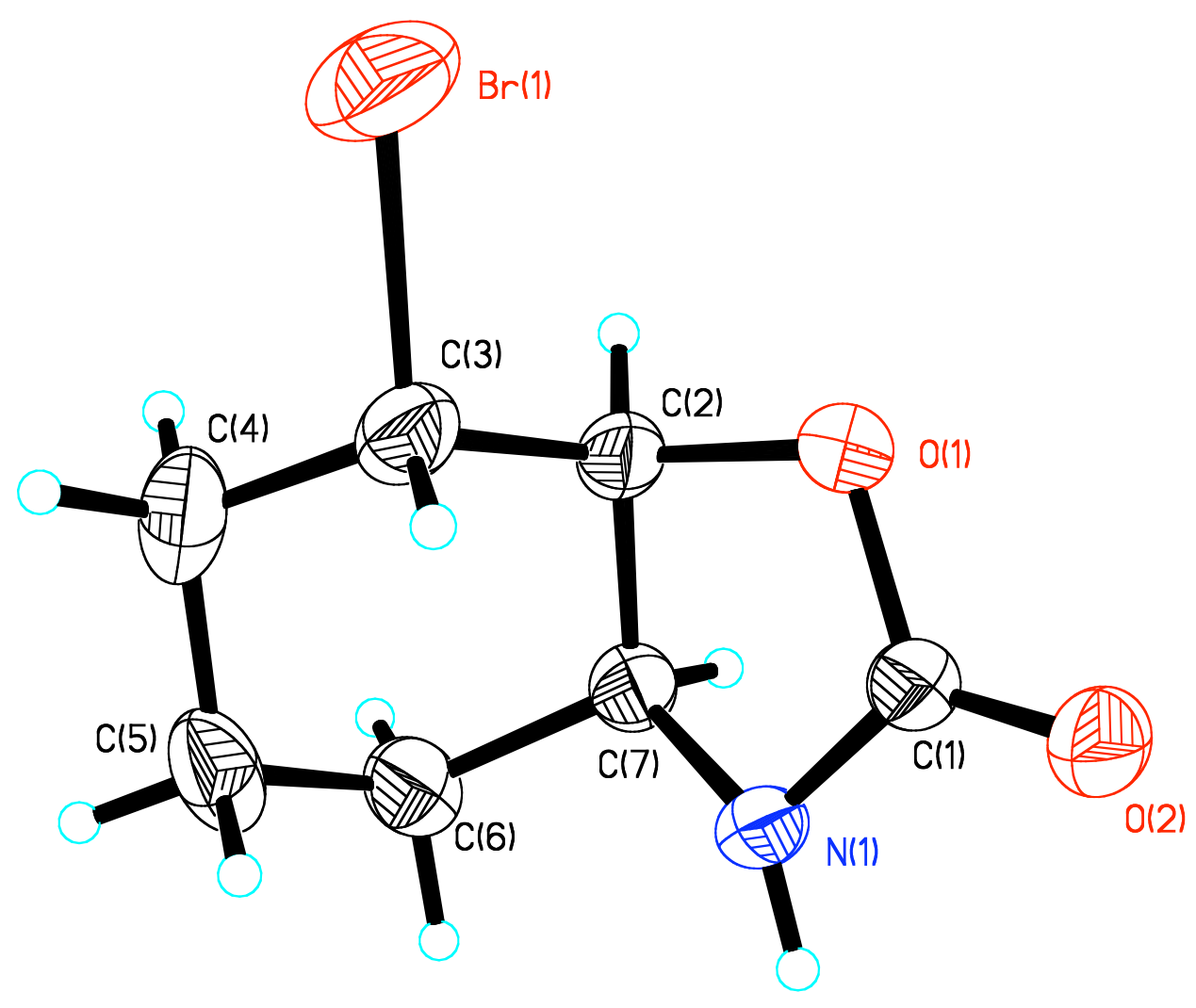

